\title{
Mutational profiles of different macroscopic subtypes of colorectal adenoma reveal distinct pathogenetic roles for KRAS, BRAF and PIK3CA
}

\author{
Li-Chun Chang ${ }^{1,2}$, Han-Mo Chiu ${ }^{3}$, Chia-Tung Shun ${ }^{4}$, Jin-Tung Liang ${ }^{5}$, Jaw-Town Linn ${ }^{3}$, Chien-Chuan Chen ${ }^{3}$, \\ Yi-Chia Lee ${ }^{3}$ and Ming-Shiang $\mathrm{Wu}^{3^{*}}$
}

\begin{abstract}
Background: Investigations of genetic alterations and correlations with histology or morphology could provide further insights into colorectal carcinogenesis. Nevertheless, such genetic changes were less investigated in adenoma stage and a comprehensive survey of oncogenic mutations in EGFR signaling pathway according to different morphologic subtypes has not been performed.

Methods: A total of 94 neoplasms, including 34 polypoid adenoma, 16 lateral spreading tumors-granular (LST-G), 20 non-granular LST (LST-NG), and 24 depressed tumors, were subjected for mutational analysis of KRAS (exon 2), BRAF (exon 11 and 15), PIK3CA (exon 9 and 20), AKT (exon 4), EGFR (exon 18-24) and HER2 (exon18-24).

Results: KRAS mutation was noted more frequently in LST (13/36, 36.1\%) than polypoid neoplasms (5/34, 14.7\%, $\mathrm{p}=0.041)$. When comparing with $L S T-N G$, LST-G had a significantly higher frequency of KRAS mutation. $(9 / 16$, $56.3 \%$ vs. $4 / 20,20.0 \%, p=0.024$ ). BRAF mutation (V600E) was found in 2 of $36(5.6 \%)$ LSTs and 1 of $34(2.9 \%)$ polypoid lesions. The two LST lesions with BRAF mutation were pathologically proven to be serrated adenoma. PIK3CA mutation (exon 9 E545K) was identified only in LST (5/36, 13.9\%). Mutations in KRAS, BRAF or PIK3CA occurred in a mutually exclusive manner. All mutations were absent in the specimens obtained from depressed type neoplasms.

Conclusions: Three different macroscopic subtypes of colorectal neoplasms display distinct carcinogenetic pathways in EGFR networking. Further molecular studies of CRCs should take macroscopic subtypes into consideration and highlight the importance of consensus and communication between endoscopic and pathologic diagnosis.
\end{abstract}

Keywords: Gene mutation, Non-polypoid colorectal neoplasm, EGFR

\section{Background}

Colorectal cancer $(\mathrm{CRC})$ is the most frequent malignancy of gastrointestinal tract. With respect to pathogenesis and therapeutic responses, CRC is regarded as a heterogenous disease. Macroscopically, CRC can be classified into polypoid and nonpolypoid subtypes [1]. The latter usually presents as flat or depressed tumors and is further subcategorized into depressed type, flat tumor less than $1.0 \mathrm{~cm}(0-\mathrm{IIa})$ and laterally spreading tumor (LST). Among nonpolypoid variants, depressed subtypes have the most aggressive behavior and represent the

\footnotetext{
* Correspondence: mingshiang@ntu.edu.tw

${ }^{3}$ Department of Internal Medicine, National Taiwan University Hospital, Taipei, Taiwan

Full list of author information is available at the end of the article
}

main lesions of de novo pathway [2-7]. Such variants are more difficult to identify by endoscopy and initially reported only in Japan. However, with the advance of image enhanced endoscopic techniques, the incidence of non-polypoid neoplasms has been rising and now accounts for $20-30 \%$ of CRC $[7,8]$. The unique clinicopathologic characteristics and biological behaviors for different macroscopic morphology suggest that distinct carcinogenetic pathways might exist for various subtypes of CRC.

The classic adenoma-carcinoma sequence of CRC was defined on histologic grounds and had been the cornerstone of current screening, surveillance and prevention. Polypoid adenoma is the main precursor lesion in such classical pathways. Serial genetic changes in APC, KRAS, 
and p53 genes had been reported in this sequence [9]. Investigations of genetic alterations and correlations with histology or morphology could provide further insights into colorectal carcinogenesis. A recent example is the identification of oncogenic mutation of BRAF in serrated pathway [10]. Furthermore, a better understanding of the molecular mechanisms underlying the development of different CRCs would open the way for patient-specific therapy. This is reflected in the current practice that antiepidermal growth factor receptor antibody therapy is no longer offered to CRC patients with mutant KRAS [11]. Moreover, nevertheless the fecal DNA testing was developed to detect significant neoplastic lesions, the precise genetic change of nonpolypoid neoplasm remains elusive and therefore such neoplasms may be missed by these tests [12]. Collectively, elucidation of genetic mechanisms by which these alterations affect colorectal carcinogenesis might have a profound impact on more effective strategies for screening, diagnosis and treatment of CRCs [13,14].

Epidermal growth factor receptor (EGFR) signaling influences multiple downstream pathways, including Ras/ Raf/MAPK and phosphatidylinositol 3'-kinase (PI3K)/ AKT pathways. Alterations of signaling in this pathway had been reported to affect cell proliferation, survival and apoptosis of a variety of malignancies [14]. A substantial portion of CRC was observed to harbor nonoverlapping somatic mutations of KRAS, BRAF, PIK3CA and AKT genes [15-23]. Nevertheless, such genetic changes were less investigated in adenoma stage and a comprehensive survey of oncogenic mutations in EGFR signaling pathway according to different morphologic subtypes has not been performed. In this study, we aimed to assess the frequency and distribution of EGFR pathway alterations in three subtypes of colorectal neoplasms. Some subcategories of 0-IIa lesions, the so called laterally spreading tumor (LST), extend laterally and circumferentially rather than vertically along the colonic wall and the frequency of invasive carcinoma is known to be less than that of polypoid lesions with similar size $[14,15]$. Though it is classified as "non-polypoid" neoplasm as a whole with depressed lesions recently, its clinicopathological characteristics are distinct from depressed (0-IIc) or flat and depressed (0-IIa + IIc or 0-IIc + IIa) lesions [6,7]. LSTs are considered as less invasive as they rarely become invasive till the size of $3 \mathrm{~cm}$ or more [6,15]. Among LSTs, granular type (LST-G) and flat type (LST-NG) are also different. Malignant transformation is more common in LST-NG at smaller size with higher risk of multifocal submucoal invasion in comparison with LST-G [15]. Some different genetic alterations were also observed between polypoid, LST-G and LST-NG [16,17]. Therefore, it is reasonable to speculate a unique biological behavior or molecular basis of malignant transformation in such non-polypoid neoplasms.
Among different phenotypes of LST, granular type and flat type were reported to have different frequency of KRAS mutation and other genetic or epigenetic changes $[16,17]$. However, concurrent analysis of KRAS and BRAF mutation for such lesions has never been conducted before. In this study, we aim to elucidate the frequency of KRAS mutation and BRAF mutation in polypoid, flat and depressed colorectal neoplasms and compare these morphological counterparts each other.

\section{Methods}

\section{Patient samples}

The study protocol was approved by institutional review board of National Taiwan University Hospital, and informed consent was obtained from all patients. We prospectively recruited 85 patients who underwent endoscopic or surgical resection of colorectal neoplasms from September 2006 to December 2008. The endoscopic morphology of colorectal tumors was mainly based on Paris and Japanese Research Society classifications with some modifications of subtypes as we have described elsewhere [1]. Lesions with 0-Is or 0-Ip were classified into "protruded" subtype (Figure 1, A \& B). LSTs were defined as lesions $\geq 10 \mathrm{~mm}$ in diameter with a low vertical axis extending laterally along the interior luminal wall. They were further subdivided into granular (LST-G) or nongranular (LST-NG) subtypes according to macroscopic findings: LST-G has even or uneven nodules on the surface whereas LST-NG type has a smooth surface (Figure 1, C \& D). Lesions categorized as 0-IIc, 0-IIc + IIa or 0-IIa + IIc were depressed subtype (Figure 1, E \& F). A total of 94 neoplasms were subjected for further analyses. These included 34 polypoid subtype, 36 LSTs (16 LST-G, 20 LST-NG), and 24 depressed subtype.

\section{Histopathological assessment}

Hematoxylin and eosin stained slides prepared from routinely processed paraffin-embedded tissue samples were assessed. The histopathological analysis of each tumor was done according to the WHO criteria [24]. Advanced colorectal neoplasia was defined as those lesions with one of the following criteria: lesions larger than $10 \mathrm{~mm}$ in diameter, lesions with a villous component, high-grade dysplastic (HGD) lesions or carcinoma in situ (CIS), and lesions with invasive features. All specimens were reviewed by the same pathologist (Chia-Tung Shun), who was unaware of the colonoscopic findings, medical history and the results of genetic analysis.

\section{DNA extraction}

DNA was extracted from surgical or endoscopic specimens. Briefly, tumor cell-rich areas in $0.1 \%$ methylene blue-stained $10-\mu \mathrm{m}$ sections under microscopy were scratched with 20 gauge needles and deparaffinized. 


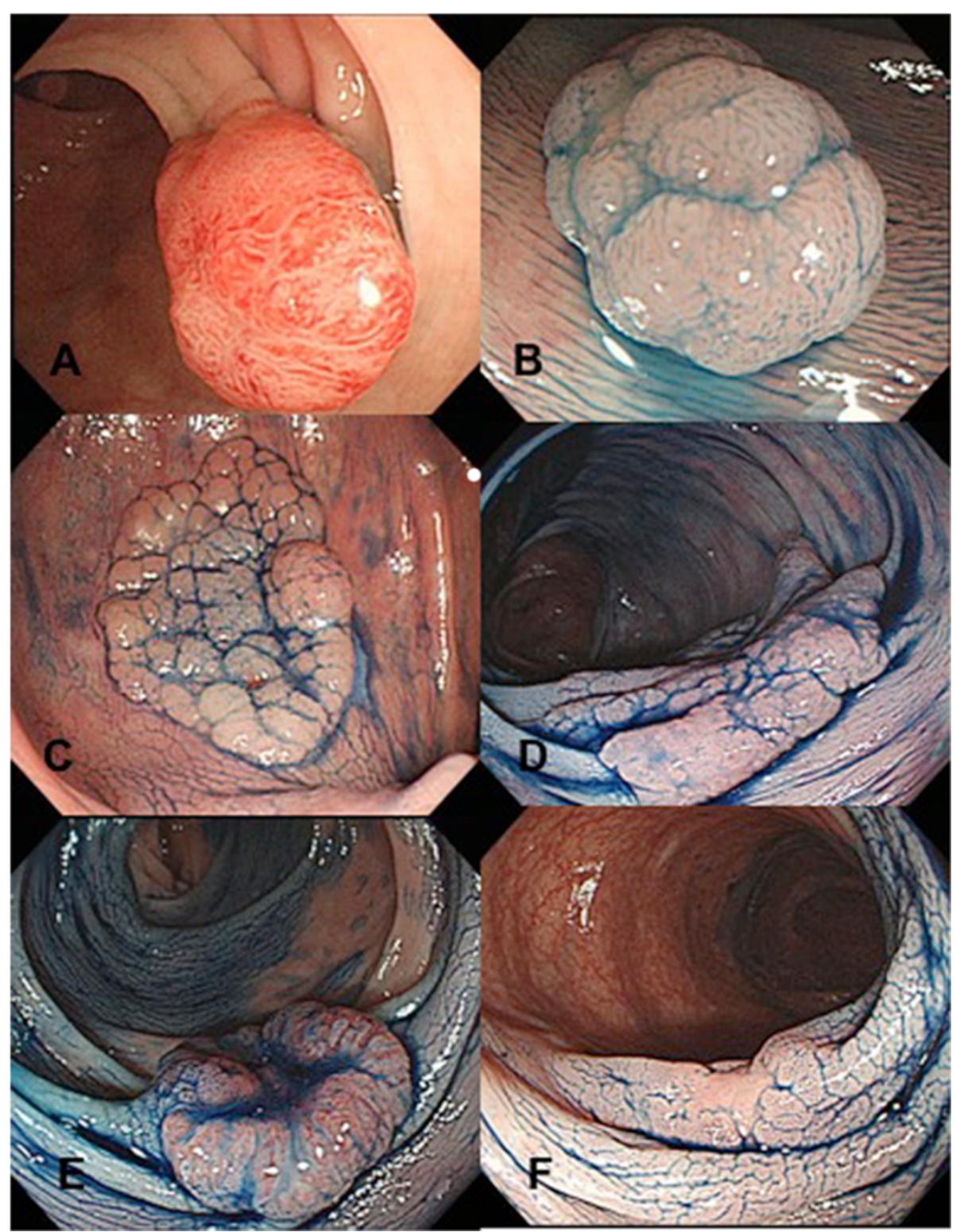

Figure 1 Macroscopic classification of colorectal neoplasm. A: 0-Ip, B: 0-Is, C: LST-G, D: LST-NG, E: 0-IIa + Ilc, F: 0-IIc.

Recovered tissues were incubated in $1 \mathrm{X}$ polymerase chain reaction (PCR) buffer containing proteinase $\mathrm{K}$ solution. After heat inactivation, the extracted DNAs were subjected for sequencing analyses.

\section{Mutation analyses}

Intron-based PCR primers were used to amplify the targeted exons of KRAS, BRAF, HER2, EGFR, PIK3CA and AKT genes based on previously published sequences. The primers were as follows (forward and reverse, respectively): BRAF exon 11 (5'TTCTGTTTGGCTTGACTTGACTT3 ' and 5'-ACTTGT CACAATGTCACCTT-3') and exon 15 (5'-TGCTTGCTCTGATAGGAAAAT G-3' and 5'-AG
CATCTCAGGGCCAAAAAT-3'); [23] KRAS exon 2 (5'CTGAAAATGACTGAATATAAACTTGT-3' and 5'-ATA TGCATATTAAAACAA GATTTACC-3'); [24] AKT exon 4 (5'-CACACCCAGTTCCTGCCT-3' and 5'-CCTGGTG GGCAAAGAGGGCT-3'); [25] PIK3CA exon 9 (5'-TCAG CAGTTACTATTCTGTGACTGG-3' and 5'-GTAAAAC GACGGCCAGTTGCTGAGATCAGCCAAATTCA-3’) and exon 20 (5'-GTAAAACGACGGCCAGTGACATTT GAGCAAAGACCTGAAG-3' and 5'-TGGATTGTGCA ATTCCTATGC-3'); [26] EGFR exon 18 (5'-AGCATGGT GAGGGCTGAGGTGAC-3' and 5'-ATATACAGCTTG CAAGGACTCTGG-3'), exon 19 (5'-CCAGATCACTGG GCAGCATGTGGCACC-3' and 5'AGCAGGGTCTAG 
AGCAGAGCAGCTGCC-3'), exon 20 (5'-GATCGCAT TCATGCGTCTTCACC-3' and 5'-TTGCTATCCCAGG AGCGCAGACC-3'), exon 21 (5'-TCAGAGCCTGGCA TGAACATGACCCTG-3' and 5'-GGTCCCTGGTGTCA GGAAAATGCTGG-3'), exon 22 (5'-AATTAGGTCCA GAGTGAGTTAAC-3' and 5'-ACTTGCATGTCAGAG GATATAATG-3'), exon 23 (5'-CATCAAGAAACAGT AACCAGTAATG-3' and 5'AAGGCCTCAGCTGTTT GGCTAAG-3'), exon 24 (5'-TTGACTGGAAGTGTCG CATCACC-3' and 5'-CATGTGACAGAACACAGTGA CATG-3'); [27] HER2 exon 18 (5'-GTGAAGTCCTCC CAGCCCGC-3' and 5'-CTCCCATCAGAACTGCCGA CC-3'), exon 19 (5'-TGGAGGACAAGTAATGATCTCC TGG-3' and 5'-AAGAGAGACCAGAGCCCAGACCTG3'), exon 20 (5'-GCCATGGCTGTGGTTTGTGATGG-3' and 5'-ATCCTAGCCCCTTGTGGACATAGG-3'), exon 21 (5'-GGACTCTTGCTGGGCATGTGG-3' and 5'-CC ACTCAGAGTTCTCCCATGG-3'), exon 22 (5'-CCATG GGAGAACTCTGAGTGG-3' and 5'-TCCCTTCACATG AGGTGG-3'), exon 23 (5'-AGACTCCTGAGCAGAAC CTCTG-3' and 5'-AGCCAGCACAGCTCAGCCAC-3'), and exon 24 (5'-ACTGTCTAGACCAGACTGGAGG-3' and 5'-GAGGGTGCTCTTAGCCACAGG-3') [28]. PCR was performed in a $20-\mu \mathrm{l}$ volume containing $100 \mathrm{ng}$ of template DNA, $10 \times$ PCR buffer; $0.25 \mathrm{mM}$ deoxynucleoside triphosphate (dNTP), 20 pmol primers, and $1.5 \mathrm{U}$ Taq DNA polymerase (Takara Shuzo, Kyoto, Japan). PCR products were electrophoresed on $2 \%$ agarose gels, purified, and both strands were directly sequenced using the BigDye Terminator v3.1 cycle sequencing kit, followed by analysis with an Applied Biosystems 3700 automated sequencer (Applied Biosystems, Foster City, CA, USA). All suspected mutations were confirmed by independent PCR amplifications and sequenced in both directions.

\section{Statistical methods}

Statistical analysis was done using SAS program (version 9.1, Cary, NC, U.S.A.). Noncontinuous variables were analyzed with chi-square test or Fischer's exact test as indicated. Continuous variables were analyzed using two-sided $t$ tests.

\section{Results}

Demographic and clinicopathological characteristics of polypoid, LST and depressed neoplasms

A total of 94 colorectal neoplasms were collected from 85 patients, including 34 polypoid lesions, 36 LST and 24 depressed lesions. Table 1 lists the demographic and clinicopathologic characteristics according to macroscopic subtypes. There was no significant difference in age and gender distribution. A higher percentage of proximal location was noted for LSTs. The tumor size was relatively smaller for depressed lesions. Severe
Table 1 Basic demographic characteristics of studied colorectal neoplasms

\begin{tabular}{lllll}
\hline & Polypoid & LST-G & LST-NG & Depressed \\
Lesion no./patient no. & $\mathbf{3 4 / 2 6}$ & $\mathbf{1 6 / 1 5}$ & $\mathbf{2 0 / 2 0}$ & $\mathbf{2 4 / 2 4}$ \\
\hline Gender(M/F) & $18 / 8$ & $6 / 9$ & $15 / 5$ & $21 / 3$ \\
Mean age & 61.0 & 67.7 & 64.9 & 61.3 \\
Proximal/distal & $16 / 18$ & $13 / 3$ & $16 / 4$ & $12 / 12$ \\
Mean size \pm SD (cm) & 1.58 & 2.21 & 1.99 & 0.89 \\
Histopathology & & & & \\
HGD & 0 & 2 & 4 & 3 \\
CIS & 0 & 2 & 3 & 0 \\
Invasive cancer & 0 & 2 & 3 & 3 \\
\hline
\end{tabular}

HGD: high-grade dysplasia; CIS: carcinoma in situ.

histological changes such as high-grade dysplasia, carcinoma in situ and invasive cancer were not noted for polypoid tumors.

\section{Mutational analyses in polypoid, LST and depressed neoplasms}

The mutational profile of all the specimens is summarized in Table 2. There was no mutation identified for EGFR, HER2 and AKT genes. KRAS mutation (codon 2) was noted more frequently in LST $(13 / 36,36.1 \%)$ than polypoid neoplasms $(5 / 34,14.7 \%, p=0.041)$. When comparing LST-G with LST-NG, LST-G had a significantly higher frequency of KRAS mutation than in LST-NG. $(9 / 16,56.3 \%$ vs. $4 / 20,20.0 \%, p=0.024)$. BRAF mutation (V600E) was found in 2 of 36 (5.6\%) LSTs and 1 of 34 (2.9\%) polypoid lesions (Table 3 ). The two LST lesions with BRAF mutation were macroscopically classified as granular type and pathologically proven to be serrated adenoma (Figure 2). In contrast, the polypoid tumor with BRAF mutation was confirmed as traditional serrated adenoma. PIK3CA mutation (exon 9 E545K) was identified only in LST $(5 / 36,13.9 \%)$. Mutations in KRAS, BRAF or PIK3CA occurred in a mutually exclusive manner. All mutations were absent in the specimens obtained from depressed type neoplasms.

Table 2 Mutation rates in different macroscopic subtypes of colorectal neoplasms

\begin{tabular}{llll}
\hline Mutation & LST(\%)(n=36) & Polypoid(\%)(n=34) & Depressed(\%)( $=\mathbf{2 4})$ \\
\hline KRAS & $13 / 36(36.1 \%)$ & $5 / 34(14.7 \%)$ & $0 / 24(0 \%)$ \\
BRAF & $2 / 36(5.6 \%)$ & $1 / 24(2.9 \%)$ & $0 / 24(0 \%)$ \\
PIK3CA & $5 / 36(13.9 \%)$ & $0 / 34(0 \%)$ & $0 / 24(0 \%)$ \\
AKT & $0 / 36(0 \%)$ & $0 / 34(0 \%)$ & $0 / 24(0 \%)$ \\
EGFR & $0 / 36(0 \%)$ & $0 / 34(0 \%)$ & $0 / 24(0 \%)$ \\
HER2 & $0 / 36(0 \%)$ & $0 / 34(0 \%)$ & $0 / 24(0 \%)$ \\
\hline
\end{tabular}


Table 3 Comparison of BRAF mutation in lesions with different macroscopic types

\begin{tabular}{llll}
\hline Morphology & BRAF $(+)$ & BRAF $(-)$ & p-value \\
\hline Polypoid $(n=34)$ & $1^{*}$ & 33 & 1.00 \\
LST $(n=36)$ & $2^{*}$ & 34 & \\
Depressed $(n=24)$ & 0 & 24 & - \\
\hline
\end{tabular}

*Pathologically turned out to have serrated change.

\section{Discussion}

A wealth of data has revealed CRCs defined by distinct molecular and pathologic features correspond with differing responses to particular chemotherapy and clinical outcomes. The increasing recognition of distinct clinicopathologic behaviors between polypoid and non-polypoid colorectal polyps had added to the need for clarification of molecular pathogenesis of these different subtypes of neoplasm. The current study represents the first comprehensive and concurrent analysis of activation mutations in EGFR network. We found flat lesions, especially granular type LST, displayed a higher frequency of KRAS and PIK3CA mutations as compared to those of polypoid tumors. Furthermore, no mutations were detected in depressed tumors, indicating existence of different molecular pathways for these clinically more aggressive lesions. Our findings support the notion that different macroscopic colorectal polyps may have distinct pathogenesis.

KRAS is a GTPase protein that is activated by EGFR and other cell surface growth factor receptors and activation of EGFR/KRAS/BRAF pathway plays a key role in the carcinogenesis of several malignancies. Mutations in KRAS can be identified in $30-40 \%$ of colorectal cancers $[13,14]$ and mutated KRAS is constitutively active independent of EGFR signaling. The presence of a KRAS mutation may predict lack of response to EGFR inhibitors in metastatic CRC $[11,13,14]$. In this study, we demonstrated that frequency of KRAS mutation was higher in LST-G than in LST-NG or polypid tumors. These findings were in agreement with the study of Sugimoto and colleagues [29]. In their study, they found LST-G displayed more frequent KRAS mutations and LST-NG had more nuclear accumulation of beta-catenin and expression of MYC. Similar observations of higher mutation rate of KRAS in LST-G have also been documented by Hiraoka and Mukawa et al. [30,31] The KRAS mutation in LST-G may vary according to different neoplasm location. Kaji et al. have reported that LST-G in the proximal colon was significantly associated with KRAS mutation [32]. Our study failed to analyze the mutation in distinct-morphology neoplasm with different location

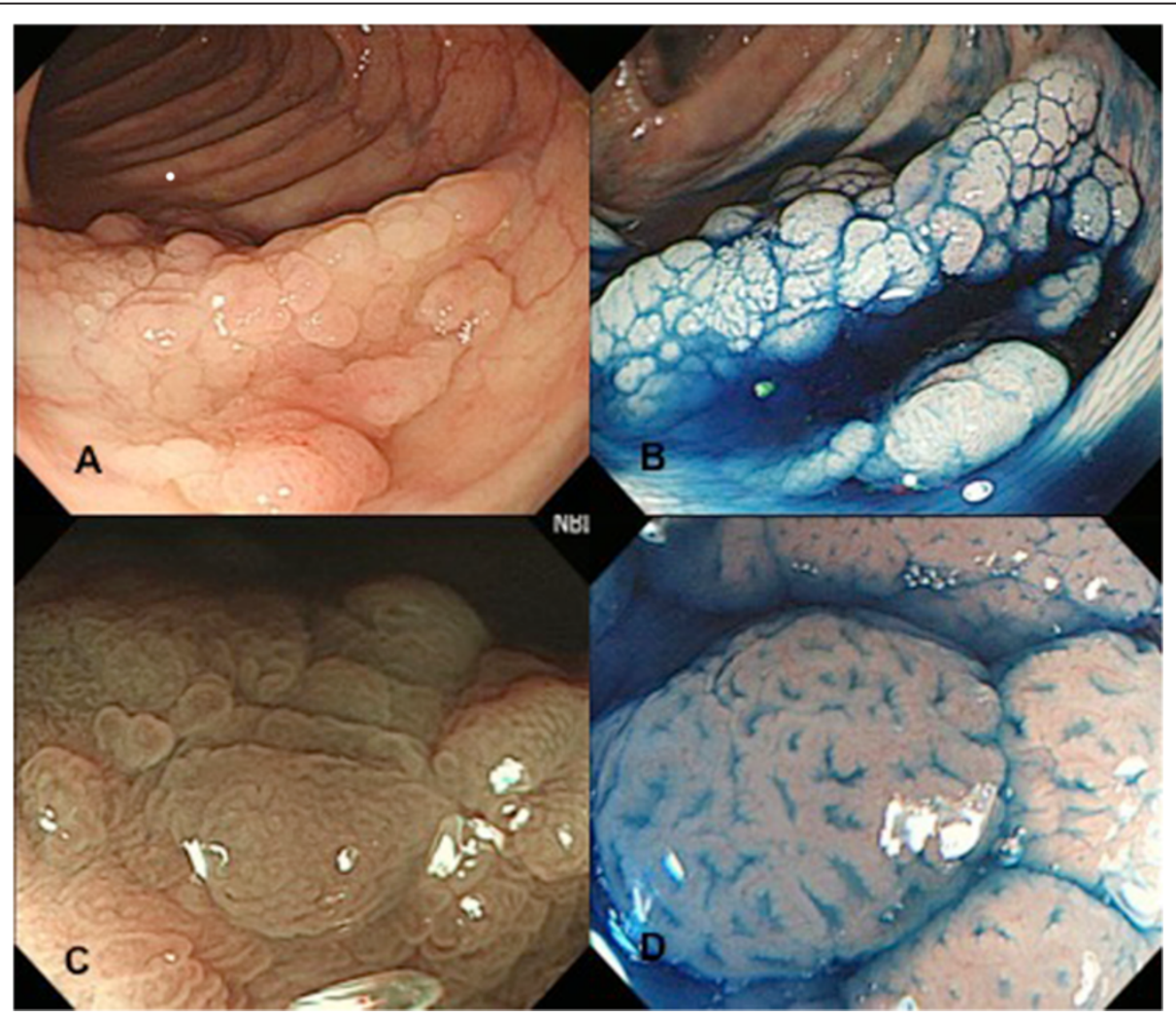

Figure 2 A case of BRAF positive LST-G. A and B: Conventional view and chromoendoscopy dye-spraying with indigo-carmine revealed 0-lla (LST-G) lesion. C: NBI with magnifying observation revealed no obvious capillary mesh. D: Magnifying observation revealed type II pit pattern. 
because of limited sample size, which is the limitation of our study. Collectively, subtypes of LST could have different molecular characteristics. Because LST in general could be detected in earlier stage and resected by endoscopy, whether these tumors, when become advanced, might have different responses to treatment remains to be further investigated.

BRAF is a downstream molecule of KRAS and mutation of BRAF V600E was detected in $5-10 \%$ of CRC $[20,23]$. Tumors with BRAF mutation are microsatellite instable, predominantly located in proximal colon, arising from serrated adenoma and have poor prognosis and unsatisfactory response to EGFR inhibitor [20,24,33]. In this study, we found two cases in LST and one case in polypoid tumors displayed BRAF mutations. Reevaluation of their pathology revealed they belonged to sessile serrated adenoma and traditional serrated adenoma respectively. Serrated pathway has recently been considered a separate one from traditional adenomaadenocarcinoma pathway because of their characteristic flat appearance and distinct molecular alterations. These tumors were more frequently overlooked by endoscopists than traditional adenomas and regarded as an important cause of interval cancer [34-36]. The increasing complexity of macroscopic and histologic classification of colorectal polyps suggested communications and consensus between endoscopists and pathologists are crucial for further investigation of molecule profiles of CRCs.

The PIK3CA gene encodes a lipid kinase regulating signaling pathway downstream of the EGFR alongside with KRAS. Mutations of PIK3CA occurred in 15-20\% of $\mathrm{CRC}$ and were associated with poor prognosis among curative resected CRCs [17,22]. The role of PIK3 mutations in determining EGFR inhibitor remains controversial. Two different reports showed discrepant results $[18,21]$. In contrast to CRC, the data of PIK3CA mutations in colorectal polyps remained few. Velho et al. have demonstrated one of 17 (5.6\%) colorectal polyps had PIK3CA mutation [16]. However, no macroscopic classification was mentioned in their study. In our study, we found PIK3CA mutations occurred predominantly in LST. Our results reemphasized the importance of macroscopic subtyping in the investigation of genetic alterations of colorectal neoplasm.

Depressed colorectal lesions, in contrast to polypoid tumors, tended to develop high-grade dysplasia or submucosal invasive cancer when they were small. The aggressive behavior and characteristic morphology suggested that they may follow a different carcinogenic pathway to flat elevated or protruding adenomas. Compared to more and more endoscopic and clinicopathologic researches for depressed colorectal tumors, investigations of genetic alterations remain scanty $[37,38]$. Previous studies for these tumors showed no mutations in KRAS and high frequencies of p53 expression by immunohistochemistry [37]. Through PCR-based pyrosequencing, Konda et al. have reported that mutation in KRAS and BRAF occurred in $16 \%$ and $11 \%$ of depressed colorectal neoplasms respectively [39]. There was a discrepancy between this study and ours. In our study, we did not find any unique genetic changes regarding EGFR network, including KRAS and BRAF, in depressed colorectal neoplasms. This discrepancy may be attributed to low tumor content among our depressed colorectal neoplasms. The lower tumor content may lead to higher false negative result. Moreover, only 3 HGD and 3 T1 cancers occurred in our total 24 depressed colorectal neoplasms. In study conducted by Konda et al., all the 19 depressed lesions harbored HGD or invasive cancers. Therefore, the more advanced histology may contribute to more genetic alterations. Finally, the sample size in both studies was limited. Only 3 and 2 depressed colorectal neoplasms had mutations in KRAS and BRAF respectively. Further researches with larger sample size and in a multi-center setting are mandatory to elucidate the relevant genetic or epigenetic changes of this special subset of CRCs.

The investigation of genetic alterations in relation to different macroscopic subtypes may also provide new insights into CRC screening. Previous studies in this field have reported the usefulness of stool DNA testing in experimental settings but only modest or unsatisfactory sensitivity for cancer and advanced adenoma were observed in population-based study [40]. Superficial neoplasms, including fat and depressed lesions, are good candidates for the target of screening and endoscopic treatment because it remains non-invasive until fairly large size $[7,41]$. Development of molecular probe with combination of variable molecular marker is an attractive field which may enable detection of such a flat neoplasm much easier [42]. Several studies have used fluorescently labeled antibodies against epitopes that are commonly overexpressed in most GI cancers, such as vascular endothelial growth factor or epidermal growth factor receptor (EGFR) [43]. If such probe for superficial colorectal neoplasm is developed, it will be of great help for improving detectability during colonoscopy. To make this scenario fulfilled, elucidations of molecular pathogenesis in different subtypes of CRC are crucial.

In summary, our findings provide further insights into the genetic alterations of colorectal neoplasms with respect to distinct macroscopic morphology. The mutational profile of KRAS, and PIK3CA in LSTs is different from protruded lesions and different subtypes of LSTs display distinct mutations. LST-G with BRAF mutation is more likely to be a sessile serrated adenoma. No specific activating mutation in EGFR network is observed in the depressed lesions although these tumors behave more aggressive than protruded adenomas and LSTs. Further 
molecular studies of CRCs should take macroscopic subtypes into consideration and highlight the importance of consensus and communication between endoscopic and pathologic diagnosis.

\section{Conclusion}

Three different macroscopic subtypes of colorectal neoplasms display distinct carcinogenetic pathways in EGFR networking. Further molecular studies of CRCs should take macroscopic subtypes into consideration and highlight the importance of consensus and communication between endoscopic and pathologic diagnosis.

\section{Abbreviations}

CRC: Colorectal cancer; LST: Laterally spreading tumor; EGFR: Epidermal growth factor receptor; LST-G: Laterally spreading tumor, granular type; LST-NG: Laterally spreading tumor, non-granular type; HGD: High-grade dysplasia; CIS: Carcinoma in situ; PCR: Polymerase chain reaction.

\section{Competing interests}

The authors declare that they have no competing interests.

\section{Authors' contributions}

L-CC drafted the manuscript. H-MC participated in concept and design of the study and carried out the generation, collection, assembly, analysis and interpretation of data. C-TS reviewed the histology critically and participated in generation of the data. J-TL carried out the concept and design of the study. J-TL carried out the molecular genetic studies and carried out the analysis and interpretation of data. C-CC participated in generation and collection of the data. Y-CL helped to performed the statistical analysis and participated in interpretation of data. M-SW participated in critical revision of the manuscript for important intellectual content. All authors read and approved the final manuscript.

\section{Acknowledgements}

Center of Excellent for Cancer Research (2014 to 2017), National Taiwan University Hospital. MOHW-103-TD-B-111-04.

\section{Author details}

'Department of Internal Medicine, National Taiwan University Hospital, Bei-Hu branch, Taipei, Taiwan. ${ }^{2}$ Graduate Institute of Clinical Medicine, College of Medicine, National Taiwan University, Taipei, Taiwan. ${ }^{3}$ Department of Internal Medicine, National Taiwan University Hospital, Taipei, Taiwan. ${ }^{4}$ Department of Pathology, National Taiwan University Hospital, Taipei, Taiwan. ${ }^{5}$ Department of Surgery, National Taiwan University Hospital, Taipei, Taiwan.

Received: 11 September 2014 Accepted: 12 December 2014 Published online: 31 December 2014

\section{References}

1. The Paris endoscopic classification of superficial neoplastic lesions: esophagus, stomach, and colon: November 30 to December 1, 2002. Gastrointest EndosC 2003,58:S3-43.

2. Fujii T, Rembacken BJ, Dixon MF, Yoshida S, Axon AT: Flat adenomas in the United Kingdom: are treatable cancers being missed? Endoscopy 1998, 30:437-443

3. Jaramillo E, Watanabe M, Slezak P, Rubio C: Flat neoplastic lesions of the colon and rectum detected by high-resolution video endoscopy and chromoscopy. Gastrointest Endosc 1995, 42:114-122.

4. Rembacken BJ, Fujii T, Cairns A, Dixon MF, Yoshida S, Chalmers DM, Axon AT: Flat and depressed colonic neoplasms: a prospective study of 1000 colonoscopies in the UK. Lancet 2000, 355:1211-1214.

5. Shimoda T, Ikegami M, Fujisaki J, Matsui T, Aizawa S, Ishikawa E: Early colorectal carcinoma with special reference to its development de novo. Cancer 1989, 64:1138-1146.
6. Tsuda S, Veress B, Toth E, Fork FT: Flat and depressed colorectal tumours in a southern Swedish population: a prospective chromoendoscopic and histopathological study. Gut 2002, 51:550-555.

7. Chiu HM, Lin JT, Chen CC, Lee YC, Laio WC, Liang JT, Shun CT, Wang HP, Wu MS: Prevalence and characteristics of nonpolypoid colorectal neoplasm in an asymptomatic and average-risk Chinese population. Clin Gastroenterol Hepatol 2009, 7:463-470.

8. Soetikno RM, Kaltenbach T, Rouse RV, Park W, Maheshwari A, Sato T, Matsui S, Friedland S: Prevalence of nonpolypoid (flat and depressed) colorectal neoplasms in asymptomatic and symptomatic adults. JAMA 2008, 299:1027-1035.

9. Takagi Y, Futamura M, Yamaguchi K, Aoki S, Takahashi T, Saji S: Genetic alterations during colorectal tumor development. N Engl J Med 1988, 319:525-532.

10. Young J, Jenkins M, Parry S, Young B, Nancarrow D, English D, Giles G, Jass J: Serrated pathway colorectal cancer in the population: genetic consideration. Gut 2007, 56:1453-1459.

11. Siena S, Sartore-Bianchi A, Di Nicolantonio F, Balfour J, Bardelli A: Biomarkers predicting clinical outcome of epidermal growth factor receptor-targeted therapy in metastatic colorectal cancer. J Nat/ Cancer Inst 2009, 101:1308-1324.

12. Imperiale TF, Ransohoff DF, Itzkowitz SH, Levin TR, Lavin P, Lidgard GP, Ahlquist DA, Berger BM: Multitarget stool DNA testing for colorectalcancer screening. N Engl J Med 2014, 370:1287-1297.

13. Cunningham D, Atkin W, Lenz HJ, Lynch HT, Minsky B, Nordlinger B, Starling N: Colorectal cancer. Lancet 2010, 375:1030-1047.

14. Lievre A, Blons $H$, Laurent-Puig P: Oncogenic mutations as predictive factors in colorectal cancer. Oncogene 2010, 29:3033-3043.

15. Minoo P, Moyer MP, Jass JR: Role of BRAF-V600E in the serrated pathway of colorectal tumorigenesis. J Pathol 2007, 212:124-133.

16. Velho S, Moutinho C, Cirnes L, Albuquerque C, Hamelin R, Schmitt F, Carneiro F, Oliveira C, Seruca R: BRAF, KRAS and PIK3CA mutations in colorectal serrated polyps and cancer: primary or secondary genetic events in colorectal carcinogenesis? BMC Cancer 2008, 8:255.

17. Barault $L$, Veyrie $N$, Jooste $V$, Lecorre $D$, Chapusot $C$, Ferraz JM, Lièvre A, Cortet M, Bouvier AM, Rat P, Roignot P, Faivre J, Laurent-Puig P, Piard F: Mutations in RAS-MAPK, PI(3)K (phosphatidylinositol-3-OH kinase) signaling network correlate with poor survival in a population-based series of colon cancers. Int J Cancer 2008, 122:2255-2259.

18. Prenen H, De Schutter J, Jacobs B, De Roock W, Biesmans B, Claes B, Lambrechts D, Van Cutsem E, Tejpar S: PIK3CA mutations are not a major determinant of resistance to the epidermal growth factor receptor inhibitor cetuximab in metastatic colorectal cancer. Clin Cancer Res 2009, 15:3184-3188.

19. Yantiss RK, Goodarzi M, Zhou XK, Rennert H, Pirog EC, Banner BF, Chen YT: Clinical, pathologic, and molecular features of early-onset colorectal carcinoma. Am J Surg Pathol 2009, 33:572-582.

20. Yokota T, Ura T, Shibata N, Takahari D, Shitara K, Nomura M, Kondo C, Mizota A, Utsunomiya S, Muro K, Yatabe Y: BRAF mutation is a powerful prognostic factor in advanced and recurrent colorectal cancer. $\mathrm{Br} J$ Cancer 2011, 104:856-862.

21. Sartore-Bianchi A, Martini M, Molinari F, Veronese S, Nichelatti M, Artale S, Di Nicolantonio F, Saletti P, De Dosso S, Mazzucchelli L, Frattini M, Siena S, Bardelli A: PIK3CA mutations in colorectal cancer are associated with clinical resistance to EGFR-targeted monoclonal antibodies. Cancer Res 2009, 69:1851-1857.

22. Ogino S, Nosho K, Kirkner GJ, Shima K, Irahara N, Kure S, Chan AT, Engelman JA, Kraft P, Cantley LC, Giovannucci EL, Fuchs CS: PIK3CA mutation is associated with poor prognosis among patients with curatively resected colon cancer. J Clin Oncol 2009, 27:1477-1484.

23. Liou JM, Wu MS, Shun CT, Chiu HM, Chen MJ, Chen CC, Wang HP, Lin JT, Liang JT: Mutations in BRAF correlate with poor survival of colorectal cancers in Chinese population. Int J Colorectal Dis 2011, 26:1387-1395.

24. Hamilton SR, Aaltonen LA: World Health Organization classifications of tumors: pathology and genetics of tumors of the digestive system. In Lyon, France: IARC; 2000.

25. Shoji K, Oda K, Nakagawa S, Hosokawa S, Nagae G, Uehara Y, Sone K, Miyamoto Y, Hiraike H, Hiraike-Wada O, Nei T, Kawana K, Kuramoto H, Aburatani H, Yano T, 
Taketani Y: The oncogenic mutation in the pleckstrin homology domain of AKT1 in endometrial carcinomas. Br J Cancer 2009, 101:145-148.

26. Kuo KT, Mao TL, Jones S, Veras E, Ayhan A, Wang TL, Glas R, Slamon D, Velculescu VE, Kuman RJ, Shih IEM: Frequent activating mutations of PIK3CA in ovarian carcinoma. Am J Pathol 2009, 174:1597-1601.

27. Shigematsu H, Lin L, Takahashi T, Nomura M, Suzuki M, Wistuba II, Fong KM, Lee H, Toyooka S, Shimizu N, Fujisawa T, Feng Z, Roth JA, Herz J, Minna JD, Gazdar AF: Clinical and biological features associated with epidermal growth factor receptor gene mutations in lung cancer. J Natl Cancer Inst 2005, 97:339-346.

28. Shigematsu H, Takahashi T, Nomura M, Majmudar K, Suzuki M, Lee H, Wistuba II, Fong KM, Toyooka S, Shimizu N, Fujisawa T, Minna JD, Gazdar AF: Somatic mutations of the HER2 kinase domain in lung adenocarcinoma. Cancer Res 2005, 65:1642-1646.

29. Sugimoto T, Ohta M, Ikenoue T, Yamada A, Tada M, Fujishiro M, Ogura K, Yamaji Y, Okamoto M, Kanai F, Kawabe T, Omata M: Macroscopic morphologic subtypes of laterally spreading colorectal tumors showing distinct molecular alterations. Int J Cancer 2010, 127:1562-1569.

30. Hiraoka S, Kato J, Tatsukawa M, Harada K, Fujita H, Morikawa T, Shiraha H, Shiratori Y: Laterally spreading type of colorectal adenoma exhibits a unique methylation phenotype and K-ras mutations. Gastroenterology 2006, 131:379-389.

31. Mukawa K, Fujii S, Takeda J, Kitajima K, Tominaga K, Chibana Y, Fujita M, Ichikawa K, Tomita S, Ono Y, Imura J, Kawamata H, Chiba T, Hiraishi $H$, Terano A, Fujimori T: Analysis of K-ras mutations and expression of cyclooxygenase- 2 and gastrin protein in laterally spreading tumors. J Gastroenterol Hepatol 2005, 20:1584-1590.

32. Kaji E, Kato J, Suzuki H, Akita M, Horii J, Saito S, Higashi R, Ishikawa S, Kuriyama M, Hiraoka S, Uraoka T, Yamamoto K: Analysis of K-ras, BRAF, and PIK3CA mutation in laterally-spreading tumors of the colorectum. $J$ Gastroenterol Hepatol 2011, 26:599-607.

33. Di Nicolantonio F, Martini M, Molinari F, Sartore-Bianchi A, Arena S, Saletti P, De Dosso S, Mazzucchelli L, Frattini M, Siena S, Bardelli A: Wild-type BRAF is required for response to panitumumab or cetuximab in metastatic colorectal cancer. J Clin Oncol 2008, 26:5705-5712.

34. Arain MA, Sawhney M, Sheikh S, Anway R, Thyagarajan B, Bond JH, Shaukat A: CIMP status of interval colon cancers: another piece to the puzzle. Am J Gastroenterol 2010, 105:1189-1195.

35. Sawhney MS, Farrar WD, Gudiseva S, Nelson DB, Lederle FA, Rector TS, Bond $\mathrm{JH}$ : Microsatellite instability in interval colon cancers. Gastroenterology 2006, 131:1700-1705.

36. Shaukat A, Arain M, Thaygarajan B, Bond JH: Is BRAF Mutation Associated with Interval Colorectal Cancers? Dig Dis Sci 2010, 55:2352-2356

37. Saito K, Arai K, Mori M: p53 overexpression and K-ras codon 12 mutations in submucosal invasive depressed-type colorectal cancer. Oncol Rep 2000, 7:741-744.

38. Morita T, Tomita N, Ohue M, Sekimoto M, Yamamoto H, Ohnishi T, Tada M, Ikenaga M, Miyake Y, Sakita I, Tamaki Y, Matsuura N, Ito M, Monden M: Molecular analysis of diminutive, flat, depressed colorectal lesions: are they precursors of polypoid adenoma or early stage carcinoma? Gastrointest Endosc 2002, 56:663-671.

39. Konda K, Konishi K, Yamochi T, Ito YM, Nozawa H, Tojo M, Shinmura K, Kogo M, Katagiri A, Kubota Y, Muramoto T, Yano Y, Kobayashi Y, Kihara T, Tagawa T, Makino R, Takimoto M, Imawari M, Yoshida H: Distinct molecular features of macroscopic subtypes of colorectal neoplasms. PLoS One 2014, 9:e103822.

40. Ahlquist DA, Sargent DJ, Loprinzi CL, Levin TR, Rex DK, Ahnen DJ, Knigge K, Lance MP, Burgart LJ, Hamilton SR, Allison JE, Lawson MJ, Devens ME, Harrington JJ, Hillman SL: Stool DNA and occult blood testing for screen detection of colorectal neoplasia. Ann Intern Med 2008, 149:441-450. W81.

41. Uraoka T, Saito Y, Matsuda T, Ikehara H, Gotoda T, Saito D, Fujii T: Endoscopic indications for endoscopic mucosal resection of laterally spreading tumours in the colorectum. Gut 2006, 55:1592-1597.

42. Goetz M, Wang TD: Molecular imaging in gastrointestinal endoscopy. Gastroenterology 2010, 138:828-833. e1.

43. Barrett T, Koyama Y, Hama Y, Ravizzini G, Shin IS, Jang BS, Paik CH, Urano Y, Choyke PL, Kobayashi H: In vivo diagnosis of epidermal growth factor receptor expression using molecular imaging with a cocktail of optically labeled monoclonal antibodies. Clin Cancer Res 2007, 13:66396648.

\section{Submit your next manuscript to BioMed Central and take full advantage of:}

- Convenient online submission

- Thorough peer review

- No space constraints or color figure charges

- Immediate publication on acceptance

- Inclusion in PubMed, CAS, Scopus and Google Scholar

- Research which is freely available for redistribution

Submit your manuscript at www.biomedcentral.com/submit
C BioMed Central 